Another application of it is very important, and that is its absorption through the skin. In many instances, it is desirable to bring out the action of the digitalis when the risk of disordering the stomach may act as a check, or even when there is already great gastric disturbance. Here it may be used by either poultices of the leaves, or flannels soaked in the infusion, or a mixture of the tincture and water applied to the abdomen and thighs. Cases are given by Christison of such use of it. Trousseau relates a very interesting case where very decided results were thus obtained (vol. i, chap. xxxv). Some cases were recently detailed in the journals of such use of it. This manner of administering digitalis should never be lost sight of, as it may now and then enable us to do what we cannot accomplish by the mouth.

Hypodermic injection is another mode of administering it which should not be overlooked. Certainly, in the experiments on frogs, this was chiefly resorted to on account of its convenience. Bouillaud pursued an endermic treatment by dusting a blister over the heart, with from six to fifteen grains of powdered digitalis. These various modes of administering dicitalis have each their advantages according to peculiar circumstances. No one plan of action must bind the practitioner's mind in fetters. He must be ready and willing to use each in its place, or even to substitute for digitalis some drug of similar action. From the known intolerance of mercury in some constitutions, and notably in those suffering from cirrhosis of the kidney, which is often followed by consequent heart-mischief, with its train of sequelæ, the old combination of digitalis, squill, and blue pill may have to be abandoned, and for the blue pill iodide of potassium substituted. Patients' prejudices, as well as their neeis, must be consulted. It is much to be desired that some drug may be discovered with equal properties to digitalis, and about whuse action no preconceived opinions, based on imperfect observation, may bias the minds of medical men. It would be easier to establisin the use of such an agent especially in this experimental age, than to war against a settled impression. And though at present the list of agrents possessing an action on the heart, and through it a control over the circulation, may be a very limited one, but at the same time very important, the day is not far distant when the importance of inquiry in this direction will make itself felt. I have not claimed for digitalis anything for which it cannot fairly substantiate its claim, nor have I accorded to it any mystical action, differing from that of any other therapeutic agrent. I have striven, as far as possible, to exercise the functions of a juilye, as well as of an advocate, and tried fairly to lay down what, in the present state of our knowledge, digitalis can do, and what it cannot. And I am quite willing to labour under the disadvantage of bein: regarded as an enthusiast, if only the subject can get that attention paid to it that it deserves; and my experience tells me in language that is unmistakeable, that the effect of agents upon the heart, of which digitalis is the most powerful one with which we are yet acquainted, can no longer be overlooked by any one in the profession who regards either his uwn interests or those of his patients.

\section{TELEOLOGY AND EVOLUTION.}

$$
\text { LY THE }
$$

REV. SAMUEL HALGHTON, M.D.Dubl, D.C.L.Oxon., F.R.S. Fellow of Trinity College, Dublin.

WITH permission, I shall take advantage of the opportunity offered by Dr. T. Clifford Allbutt's letter of the I 2 th instant, published in the BRITISH MEDICAL JOURNAL of the 15 th instant, to make a few brief remarks on Telcolosy and livolution.

There is no necessary opposition between these expressions; and it is quite possible, perhaps even probable, that Teleology works out its purpose by means of livolution. If the Evolution be the result of foresight and contrivance, it becomes merely the expression of the chain of Second Causes by means of which the purposes of the great First Cause are harmoviously carried out. But if, on the other hand, Evolution admit of chance combinations, capable of becoming permanent, and not foreseen, then it is irreconcileable with the fundamental hypothesis of Teleologr:

Dr. Allbutt has, I believe, correctly interpreted what I intended to show in my accasiunal references to Teleology during my lectures on the Principle of Lea:t Action. I illustrated this principle by means of the limbs of the tiger and the wing of the albatross, and endeavoured to show that the several conditions which combine to produce the action of those members are so related to each other as to fulfil the requirements of the principle of Least Action; or, in other words, that these conditions are so related to each other that, if all of them save one be given, we can calculate with certainty the remaining condition.

This remarkable fact is, in my judgment, irreconcileable with the hypothesis of Evolution, by means of slow variation of the conditions, which is the fundamental idea of Mr. Darwin's hypothesis.

Let us suppose, in fact, that the exercise of any complex organ of an animal depends upon a certain number of conditions, which we may call $a, b, c$, etc.; then the result may be expressed as a function of those conditions,

$$
F\left(a, b, c \text {, etc., } x, y^{\prime}, c\right) \text {. }
$$

The principle of Least Action requires this function to be a maxi. mum, which will be the case for certain definite groups of values of the elementary conditions on which the complex function depends, and for no others. We shall, therefore, have the principle of Least Action fulfilled for certain groups of values.

$$
F(a, b, c \text {, etc., } x, y, s) \text {. }
$$

$$
F^{\prime}\left(a^{\prime}, b^{\prime}, c^{\prime} \text {, etc., } x^{\prime}, y^{\prime}, z^{\prime}\right) .- \text { And so on. }
$$

I showed in my lectures that, if certain conditions, $a, b, c$, etc., be given, the other unknown conditions, $x, y, z$, can be calculated on the hypothesis of Least Action; and I verified this statement by several remarkable examples. We may therefore consider it as proved, or at least admitted, that the existing arrangements of Nature fulfil the conditions imposed by the principle of Least Action. This admission is fatal to the hypothesis of Evolution by slow variation. For, let us suppose the two functions $F$ and $F^{\prime}$ each to satisfy the conditions of Least Action, each of these will represent a complex result which is stable, and which will refuse to vary indefinitely from its original value. Let the quantities $x, y, z$, etc., vary by slow degrees in any direction whatever, I assert that the principle of Least Action, which requires the function $F$ to be a maximum, will prevent $x, y, z$ from varying indefinitely ; or, in other words, that the controlling influence of the remaining conditions, $a, b, c$, etc., will compel $x, y^{\prime}, z$ to return back to their original values; which values, in conjunction with those of $a, b, c$, etc., rendered the function $F$ a maximum. In like manner, $F^{\prime}$ is another maximum ; and its elements, $a^{\prime}, b^{\prime}, \ldots x^{\prime}, y^{\prime}$, etc., cammot vary indefi. nitely by slow degrees.

The conditions involved in $F$ and $F^{\prime}$ are each stable; and, when variations of small extent occur, there is a necessary return to the condition $F$, or $F^{\prime}$, which involves the maximum effect; but it is absolutely impossible for $F$ to be transformed into $F^{\prime}$ by slow variations of its elements, without losing that property of being a maximum on which its continued existence depends.

The function $F$ is a maximum only for certain definite, and often far removed, groups of values of $a, b, c$, etc., $x, y, z$; and we cannot transform $F$ into $F^{v}$ without making the hypothesis of abrupt changes in the values of the elements, for any small slow changes will reproduce $F$ again and again.

The force of this argument will remain unchanged, if by $F$ and $F^{\prime}$ we mean two species, instead of two arrangements of organs.

An illustration from physical astronomy will clear up this point. If there were only one planet revolving round the sun, it would describe an ellipse which would remain the same at each revolution; but, as there are several planets, each exerting a small disturbing force, the planet describes an ellipse which differs slightly at each revolution from its predecessor and from its successor. Still the path is always an ellipse, and never shows any tendency to become an hyperbola or para. bola, for the simple reason that all its elements change slowly. Let us now, however, take the case of a comet, moving in an hyperbolic path round the sun, and coming into close proximity to a large planet: the elements of the comet's path, $a, b, c$, etc., are now abruttly changed, and we find the hyperbolic path changed in a few hours into an ellipse.

The Evolution of one species from another must, in my opinion, be the result of sudden, not of gradual, changes of conditions; and no scientific attempt has as yet been mace to show how such evolution of species may take place.

The concluding portion of Dr. Allbutt's letter, respecting the surplasage of force sometimes found in Nature, opens up a subject of discussion on which I could say much, were I not afraid to weary by my much writing, as I have already offended some by my much talking.

In conclusion, I would remark that, in the supposed slow passage of an organ or species from the maximum $F$ to another maximum $F^{\prime}$, it must necessarily pass through a minimum, which will exhibit in an extreme degree want of contrivance and imperfection. Where are these imperfections? They ought vastly to exceed in number the perfect types which have reached the maximum effect. Where is the evidence of the endless succession of blunders which blind Nature has used as stepping-stones to produce her few happy successes? I cannot find them either in living nature or in the records of palieontology. 